\title{
Dynamics of filament formation in a Kerr medium
}

\author{
Martin Centurion, ${ }^{1} \mathrm{Ye} \mathrm{Pu},{ }^{2}$ Mankei Tsang, ${ }^{2}$ and Demetri Psaltis ${ }^{2}$ \\ ${ }^{1}$ Physics Department, California Institute of Technology, Pasadena, California 91125, USA \\ ${ }^{2}$ Department of Electrical Engineering, California Institute of Technology, Pasadena, California 91125, USA
}

(Received 2 December 2004; published 17 June 2005)

\begin{abstract}
We have studied the large-scale beam breakup and filamentation of femtosecond pulses in a Kerr medium. We have experimentally monitored the formation of stable light filaments, conical emission, and interactions between filaments. Three major stages lead to the formation of stable light filaments: First the beam breaks up into a pattern of connected lines (constellation), then filaments form on the constellations, and finally the filaments release a fraction of their energy through conical emission. We observed a phase transition to a faster filamentation rate at the onset of conical emission. We attribute this to the interaction of conical emissions with the constellation which creates additional filaments. Numerical simulations show good agreement with the experimental results.
\end{abstract}

DOI: 10.1103/PhysRevA.71.063811

PACS number(s): 42.65.Jx, 42.65.Tg, 89.75.Kd

\section{INTRODUCTION}

The formation of optical filaments when high-power laser pulses propagate in Kerr media has generated widespread interest since the phenomenon was first observed [1-3]. When the laser power is much higher than the critical power for self-focusing, an initially uniform beam breaks up into small filaments [4,5]. Recently, the breakup of high-power femtosecond pulses in air into multiple filaments was reported [6]. The formation of small-scale filaments has also been observed in glass [7]. Here we present an extensive study of large-scale beam filamentation of femtosecond pulses in carbon disulfide $\left(\mathrm{CS}_{2}\right)$. The spatial evolution of the beam was studied as a function of propagation distance and energy. We have observed three phases leading to the formation of steady-state filaments (solitary waves $[8,9]$ ). The three stages are constellation formation, filamentation, and conical emission. The mechanism for beam breakup is modulation instability of spatial modes $[10,11]$. It has been shown that for multiple filamentation, the power trapped in each filament is constant and does not depend on the power of the input beam $[4,10]$. Thus, the number of filaments is expected to increase linearly with input power.

We have observed a phase transition in the rate of filament formation with input power which we attribute to the observed conical emissions from individual filaments. We have observed such conical emissions from individual filaments in $\mathrm{CS}_{2}$ lasting less than 2 ps typically when the filaments first approach their minimum diameter. The result is that a fraction of the energy trapped in the filament is released, while the central component continues to propagate with a broadened spectrum. Conical emissions similar to the ones we have observed were theoretically predicted by Luther et al. [12]. It is believed that conical emissions are also tied to temporal pulse splitting and spectral broadening [13-15] since they occur shortly after the filament nears collapse to a very small area. Conical emissions from individual filaments of a different physical origin were experimentally measured for nanosecond pulses propagating through atomic vapors [16] and femtosecond pulses propagating in air
$[17,18]$. In the experiment we describe in this paper, we were able to generate and track a large number of filaments $(>100)$. This allowed us to measure the statistics of conical emissions and capture filament interactions such as spatial splitting and fusion [19-22]. Our experimental results are complemented with computer simulations of the spatial evolution of the beam and the propagation of single self-trapped filaments.

\section{EXPERIMENTAL SETUP}

A schematic diagram of the experimental setup is shown in Fig. 1. The Ti:sapphire amplified laser system generates 150 -fs pulses with a central wavelength of $800 \mathrm{~nm}$ at a repetition rate of $10 \mathrm{~Hz}$. The maximum pulse energy was $2 \mathrm{~mJ}$, with a shot-to-shot variation of $3 \%$. The duration of the pulses is kept constant throughout the experiments, while the energy is adjusted as indicated. The beam profile was approximately Gaussian with a $5 \mathrm{~mm}$ full width at half maximum (FWHM). $\mathrm{CS}_{2}$ was chosen as the nonlinear material because of its strong Kerr nonlinearity. A value of $n_{2}=3$ $\times 10^{-15} \mathrm{~cm}^{2} / \mathrm{W}$ has previously been measured in $\mathrm{CS}_{2}$ using femtosecond pulses [23]. A glass cuvette with a $10 \mathrm{~mm}$ path length is filled with $\mathrm{CS}_{2}$. The nonlinear pulse propagation was monitored by imaging the beam profile for different input pulse energies and different propagation lengths.

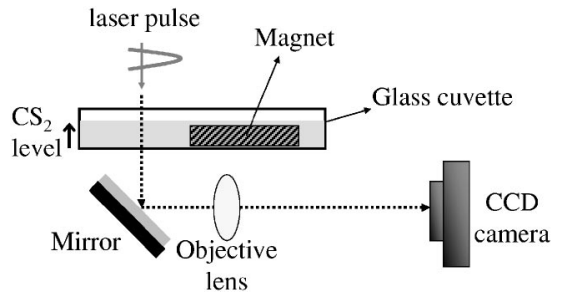

FIG. 1. Experimental setup. A femtosecond pulse traverses a glass cell filled with carbon disulfide. The beam profile at the output of the cell is imaged on a CCD camera. A magnet inside the cell is moved to adjust the liquid level, which determines the propagation distance. 


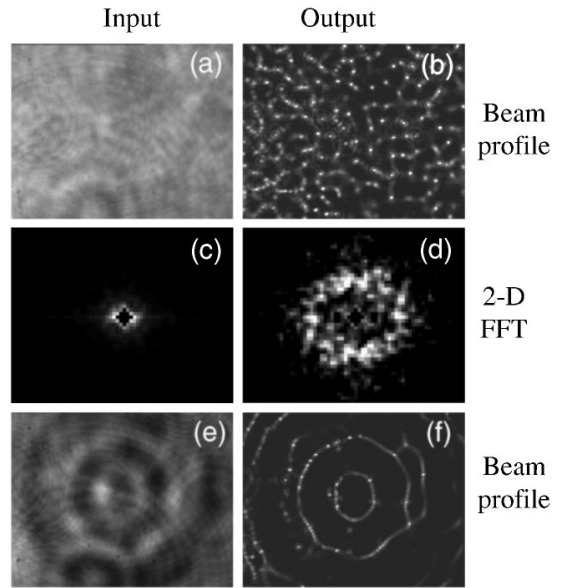

FIG. 2. Beam profile and 2D fast Fourier transform (FFT) before and after traversing $10 \mathrm{~mm}$ of $\mathrm{CS}_{2}$. (a) Input beam profile. (b) Beam profile after traversing $10 \mathrm{~mm}$ of $\mathrm{CS}_{2}$. (c) and (d) are the 2D FFT of (a) and (b), respectively. (e) and (f) are the input and output beam profiles for a modulated beam.

The laser beam is redirected so that it propagates downward and into the glass cell. The liquid level in the cuvette determines the propagation length. A pair of magnets is used to adjust the liquid level in situ. One of the magnets lies inside the cell and can be controlled by moving the second magnet outside. The liquid level was adjusted from 5.5 to $8 \mathrm{~mm}$ by submerging or lifting the inside magnet, with a minimum step size of $0.25 \mathrm{~mm}$. It is very important to change the propagation length in situ as any changes in the beam profile or position of the cell can lead to different filamentation patterns. With our setup we are able to repeatedly generate the same patterns and identify and follow the propagation of individual filaments. The beam profile that forms at the bottom of the cell is imaged onto a high-resolution charge-coupled device (CCD) camera (Apogee AP32ME, $2184 \times 1472$ pixels, pixel size $6.8 \mu \mathrm{m})$. The image is magnified by a factor of 5 using a $5 \times$ microscope objective (numerical aperture 0.1 , focal length $25.4 \mathrm{~mm}$ ). The light is attenuated by a factor of $4 \times 10^{-5}$ using a neutral density filter in front of the camera. The camera is triggered by the laser and each image is obtained with a single laser pulse.

\section{CONSTELLATION FORMATION AND FILAMENTATION}

\section{A. Experimental results}

As the beam propagates in the nonlinear medium we observed an initial formation of a pattern of connected lines (constellation). As the pulse propagates further, filaments form on the constellation. Figure 2(a) shows a picture of the input beam profile. Figure 2(b) shows the beam profile for a $0.78-\mathrm{mJ}$ pulse after traversing $10 \mathrm{~mm}$ of $\mathrm{CS}_{2}$. Figures 2 (c) and 2(d) show the two-dimensional (2D) Fourier transforms of the beam profile before and after the nonlinear interaction (the central component was blocked for visual clarity). The ring observed in the Fourier plane is due to the self-phasemodulation of an extended beam propagating in a $\chi^{(3)}$ me-

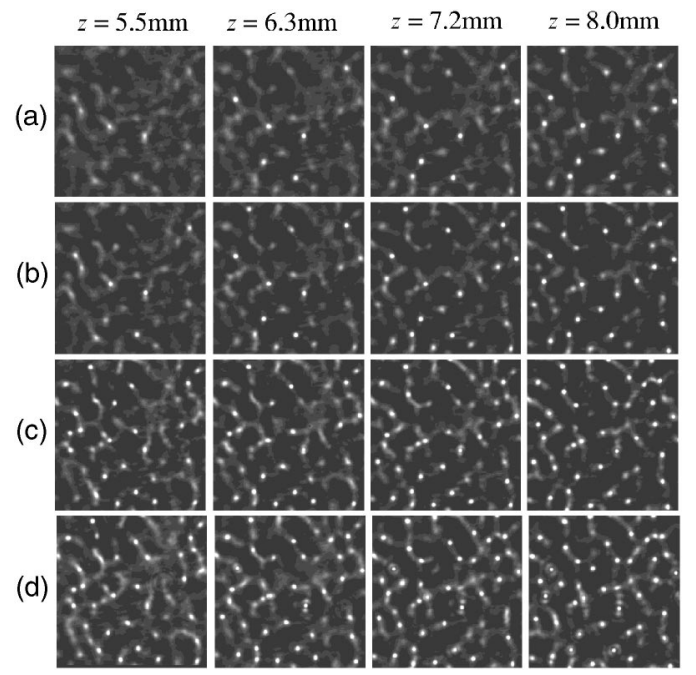

FIG. 3. Beam breakup and filamentation as a function of propagation distance (left to right) and energy (top to bottom). $E=$ (a) 0.70 , (b) 0.86 , (c) 1.0 , and (d) $1.1 \mathrm{~mJ}$. The size of each image is $0.5 \times 0.5 \mathrm{~mm}^{2}$.

dium. The light is redirected preferentially in a particular direction, due to the phase-matching condition necessary for efficient scattering. We will discuss this in detail in the following section.

Once the constellation is formed it remains stable as the beam continues to propagate (within our observation range), except for the appearance of an increasing number of bright spots (filaments). The width of the stripes in the constellation decreases until it reaches a minimum size of $15-20 \mu \mathrm{m}$, after which the width remains constant and the constellation continues to breakup into filaments. The constellation remains unchanged as the pulse energy is increased from 0.5 to $1.1 \mathrm{~mJ}$. The only change we observed with increased pulse energy is the formation of additional filaments. The constellation pattern is very sensitive to the input beam profile. For example, the beam can be induced to form a constellation in a specified pattern. Figures 2(e) and 2(f) show the beam profile before and after the nonlinear propagation for a beam that was passed through a circular aperture to generate diffraction rings. After traversing the $\mathrm{CS}_{2}$ cell the beam breaks up in a circular pattern resembling that of the input beam.

Figure 3 shows the evolution of the beam with propagation distance for four different energy levels $[0.70$ (a), 0.86 (b), $1.0(\mathrm{c})$, and $1.1 \mathrm{~mJ}(\mathrm{~d})]$. A fraction of the output beam profile $\left(0.5 \times 0.5 \mathrm{~mm}^{2}\right)$ is shown for propagation distances of 5.5, 6.3, 7.2, and $8.0 \mathrm{~mm}$. The input beam profile [Fig. 4(a)] is kept constant throughout the experiment. The number of filaments is seen to increase with both energy and propagation distance, while the constellation remains fixed. Most of the filaments are stable and once they are formed they survive as the propagation length or energy is increased (within the limits of the experiment). The filaments propagate with a stable diameter of $11.8 \pm 2.6 \mu \mathrm{m}$ (FWHM) for at least $3 \mathrm{~mm}$. When the density of filaments becomes high, interactions between the filaments cause the whole pattern to become unstable (it changes drastically from shot to shot even if input parameters are the same). 
Input beam

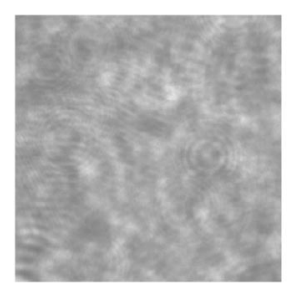

(a)

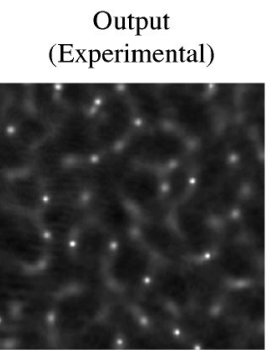

(b)

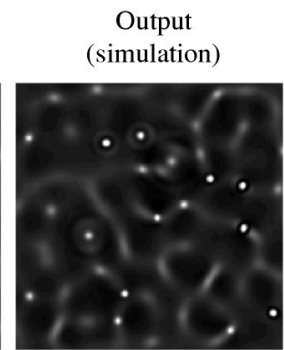

(c)
FIG. 4. The amplitude of the input beam (a) was measured and used as the input of the numerical simulation. There is good agreement between the experimental (b) and simulated (c) output beam profile. The images are $0.5 \times 0.5 \mathrm{~mm}^{2}$.

For an input energy of $0.70 \mathrm{~mJ}$ [Fig. 3(a)], at a propagation distance of $5.5 \mathrm{~mm}$ the constellation has already formed but no filaments are observed. As the pulse continues to propagate filaments start to appear in increasing number within the constellation. After the filaments reach a minimum size, conical emissions can be observed as a ring around the filaments [Figs. 3(c) and 3(d), $z=8 \mathrm{~mm}$ ]. We have also observed spatial splitting and fusion of filaments (see Sec. V). It is interesting to note that the effects of increasing the energy or the propagation length are similar. It is possible to find images across Fig. 3 with different energy and propagation distance with roughly the same number of filaments, for example $E=0.70 \mathrm{~mJ}, d=7.2 \mathrm{~mm}$ and $E=0.86 \mathrm{~mJ}, d$ $=6.3 \mathrm{~mm}$. The similarity is due to the fact that increasing the energy speeds up the self-focusing process that generates the filaments. This is only expected to hold for some range of energies and distances. For longer propagation distance we have observed the number of filaments to reach saturation and eventually start to decrease.

The Kerr effect $\left(n_{2}\right)$ and diffraction alone cannot generate stable light filaments. Another nonlinearity must act to stop the self-focusing. The size of the filaments is an indication of the strength of this nonlinearity. It is known that the addition of a saturation mechanism in the third-order nonlinearity such as fifth-order nonlinearity will generate stable filaments [24]. We have included a negative $n_{4}$ (fifth-order nonlinearity) in our simulations and seen good agreement between simulations and experiments.

\section{B. Simulations and discussion}

We will first discuss the formation of the constellations. The dominant spatial frequency of the constellations [the diameter of the ring in Fig. 2(d)] is determined by a nonlinear phase-matching condition [10] and the input beam profile. As a way to illustrate the phase-matching condition, assume we start with a uniform pump beam propagating through a Kerr medium, with a small fraction of the beam energy being scattered in all directions. The presence of the nonlinear index change will cause the strong pump beam to be phase matched to waves scattered at a specific angle. Assuming the pump beam remains undepleted, one can derive a simple relation for the transverse wave vector with the maximum nonlinear gain [10]

$$
q=k \sqrt{\frac{2 n_{2} I}{n_{0}}},
$$

where $q$ is the transverse wave vector, $I$ the intensity of the beam, $n_{0}$ the index of refraction of the material, and $n_{2}$ the Kerr coefficient.

The phase-matching condition depends on the intensity of the beam. However, in our experiments the constellations did not change as the energy was increased. We believe there are two reasons for this discrepancy. First, in the experiments the input beam was not entirely smooth. Therefore, a spatial frequency initially dominant in the beam will be amplified the most and a small change in the gain spectrum (through a small change in the intensity) will not necessarily result in a different constellation. A similar result was reported by Abbi et al. [25] who showed that for a small number of filaments, the position of the filaments at the output was correlated to weak modulation of an approximately uniform input beam. The second reason is that the phase-matching condition exists only when there is a well-defined (undepleted) pump beam. For higher intensity levels the beam quickly breaks up and the phase-matching condition no longer holds.

After the constellations are formed, the self-focusing process acts locally to break up the constellation lines into selftrapped light filaments (hot spots). Filaments form at the local maxima of the intensity distribution in the constellation. The formation and propagation of the filaments is discussed in detail in the following sections. We now compare the experimental results with a numerical simulation.

A complete simulation of the spatial and temporal profile of the nonlinear pulse propagation requires very fine sampling in three spatial dimensions and time. The light propagation is calculated assuming a scalar envelope for the electric field, which is slowly varying in time and along the propagation direction $z$. The evolution of the scalar envelope is given by the equation

$$
\begin{aligned}
\frac{\partial A}{\partial z}= & \frac{i}{2 k n_{0}}\left(\frac{\partial^{2}}{\partial x^{2}}+\frac{\partial^{2}}{\partial y^{2}}\right) A+i k\left(n_{2}|A|^{2}\right) A+i k\left(n_{4}|A|^{4}\right) A \\
& -\frac{j \beta_{2}}{2} \frac{\partial^{2} A}{\partial T^{2}} .
\end{aligned}
$$

$A(x, y, z, T)$ is the complex envelope of the electric field, $k$ $=2 \pi / \lambda, \lambda=800 \mathrm{~nm}, n_{0}=1.6, n_{2}=3 \times 10^{-15} \mathrm{~cm}^{2} / \mathrm{W}[23], n_{4}$ $=-2 \times 10^{-27} \mathrm{~cm}^{4} / \mathrm{W}^{2}, \beta_{2}=200 \mathrm{ps}^{2} / \mathrm{km}$ [26], and $T$ is the time measured in the reference frame moving at the group velocity of the pulse. The first term on the right-hand side accounts for diffraction, the second is Kerr self-focusing (third-order nonlinearity), the third term accounts for the fifth-order nonlinearity, and the last term accounts for dispersion. A linear absorption term was not included because $\mathrm{CS}_{2}$ is highly transparent at $800 \mathrm{~nm}$; no intensity changes were measured for a propagation distance of $10 \mathrm{~mm}$. Two-photon absorption has been measured in $\mathrm{CS}_{2}$ with a value of $(4.5 \pm 1) \times 10^{-13} \mathrm{~cm} / \mathrm{W}$ [27]. We have tried including this term in the simulation and did not see any significant changes in the output.

Previous simulations of femtosecond pulse propagation through different materials have included plasma generation 


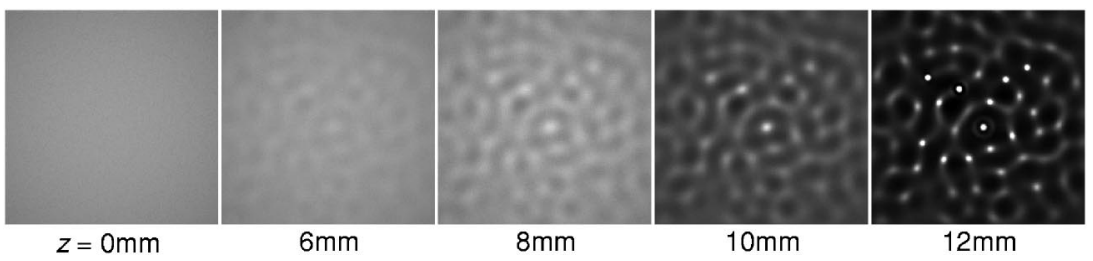

FIG. 5. Numerical simulation of beam propagation. A constellation and filaments are generated from an input beam with random amplitude noise. The images show the beam profile for propagation distances of $0,6,8,10$, and $12 \mathrm{~mm}$.

as a mechanism to arrest the collapse of the optical filaments $[17,28-30]$. The high intensity of the pulses causes ionization of the material and the generated electron plasma contributes a negative index change which balances the positive index change due to the Kerr effect. In our experiments the intensity of the filaments is not sufficient to ionize the material. To verify this we have calculated, following the Keldysh method [31,32], the intensity threshold for breakdown in $\mathrm{CS}_{2}$ to be $2 \times 10^{13} \mathrm{~W} / \mathrm{cm}^{2}$ (the ionization energy of $\mathrm{CS}_{2}$ is $10.08 \mathrm{eV}$ [33]). This calculation method has been previously compared with experimental results for a wide range of parameters and it was shown to be accurate within a factor of 2 $[34,35]$. Using the CCD camera, we have measured the energy trapped in the filaments to be $0.1 \pm 0.04 \mu \mathrm{J}$. For the measured energy, the measured filament size, and a pulse duration of $150 \mathrm{fs}$, the intensity of the filaments is $(5.9 \pm 2.4) \times 10^{11} \mathrm{~W} / \mathrm{cm}^{2}$, which is more than 30 times smaller than the calculated breakdown threshold. This justifies our conclusion that plasma generation does not play a significant role in the propagation through $\mathrm{CS}_{2}$. It has been shown that including nonparaxial effects in the simulation can also prevent the collapse of the filaments [36]. However, nonparaxiality becomes significant only when the diameter of the filaments approaches the wavelength and we have measured a minimum filament diameter of approximately $12 \mu \mathrm{m}, 15$ times larger than the wavelength. We have included the fifth-order term in the nonlinear polarization $\left(n_{4}\right)$ as the only logical remaining mechanism to balance the selffocusing and generate stable light filaments. We were not able to find published measurements of the value of $n_{4}$, and therefore we used in the simulations the value that gave us the best match with experiment.

Instead of calculating the full temporal and spatial evolution of the light field, we have used a simpler model which captures the spatial evolution of the beam, where we assume that the temporal profile of the pulse remains constant:

$$
\frac{d A}{d z}=\frac{i}{2 k n_{0}}\left(\frac{\partial^{2}}{\partial x^{2}}+\frac{\partial^{2}}{\partial y^{2}}\right) A+i k\left(n_{2}|A|^{2}\right) A+i k\left(n_{4}|A|^{4}\right) A .
$$

In this case $A(x, y, z)$ is a function of the spatial coordinates only. The fifth-order nonlinearity is introduced to generate stable light filaments, as observed in the experiments. The equation is solved using the split-step Fourier method [37]. In these experiments the changes in the temporal profile of the pulse do not play a major role in the spatial evolution of the beam. We have simulated the propagation of a single filament including the temporal profile and seen that the pulse duration does not change significantly for the experimental propagation distance (see Sec. V B).

The intensity distribution of the beam before traversing the nonlinear material was captured using a CCD camera [Fig. 4(a)]. The square root of the laser beam profile captured on the CCD camera was used as the amplitude of the input light field for the simulation. The phase of the input light was assumed to be spatially uniform. In the experiment, a pulse (0.8 mJ, $150 \mathrm{fs}, 5 \mathrm{~mm}$ FWHM) propagates in $\mathrm{CS}_{2}$ for a distance of $8 \mathrm{~mm}$. The simulation is run for the same intensity and propagation length as in the experiment. Figure 4 shows a comparison of the experimental and numerical results. The simulated beam also breaks up into constellations and filaments similar to those observed in the experiment. The simulation does not reproduce the experimental results exactly, i.e., the positions of the filaments are different. This is due to the lack of phase information of the input beam profile and possibly due to other nonlinearities not included in the simulation. However, the simulation clearly captures the main features of the propagation phenomena.

Even though the constellation pattern depends on to the input beam profile, the formation of a constellation does not require a specific spatial modulation of the input. It has been shown theoretically that any amplitude perturbation of the input light will cause the beam to break up [4]. In order to demonstrate this and study the dependence of the constellation pattern on the intensity of the input beam, we have simulated the propagation of a Gaussian beam with random noise in its amplitude. Spatially random noise is generated on the computer and superimposed on the amplitude of the input beam. The standard deviation of the noise pattern is $3 \%$ of the beam amplitude. Figure 5 shows the simulation results for a beam with an initial intensity of $3.1 \times 10^{10} \mathrm{~W} / \mathrm{cm}^{2}$ and a beam diameter of $1.6 \mathrm{~mm}$. After propagating for $6 \mathrm{~mm}$ some weak amplitude modulation is observed as the constellations start to form [Fig. 5(b)]. From 6 to $10 \mathrm{~mm}$ [Figs. 5(c) and $5(\mathrm{~d})$ ] more light is focused in the constellations and the background level is reduced. Only at a propagation distance of $12 \mathrm{~mm}$ [Fig. 5(e)] do filaments start to appear, as opposed to $8 \mathrm{~mm}$ when the experimental beam profile is used as the input. This simulation shows that the constellation can grow out of an input with only random noise. We repeated this simulation for different values of the input intensity and saw an increase in the spatial frequency of the constellations with input beam intensity for intensities below $2 \times 10^{10} \mathrm{~W} / \mathrm{cm}^{2}$. In this case the constellations grew out of random noise, so the dominant spatial frequency was determined only by the nonlinear phase-matching condition. However, as the power was increased above this value the beam was rapidly de- 

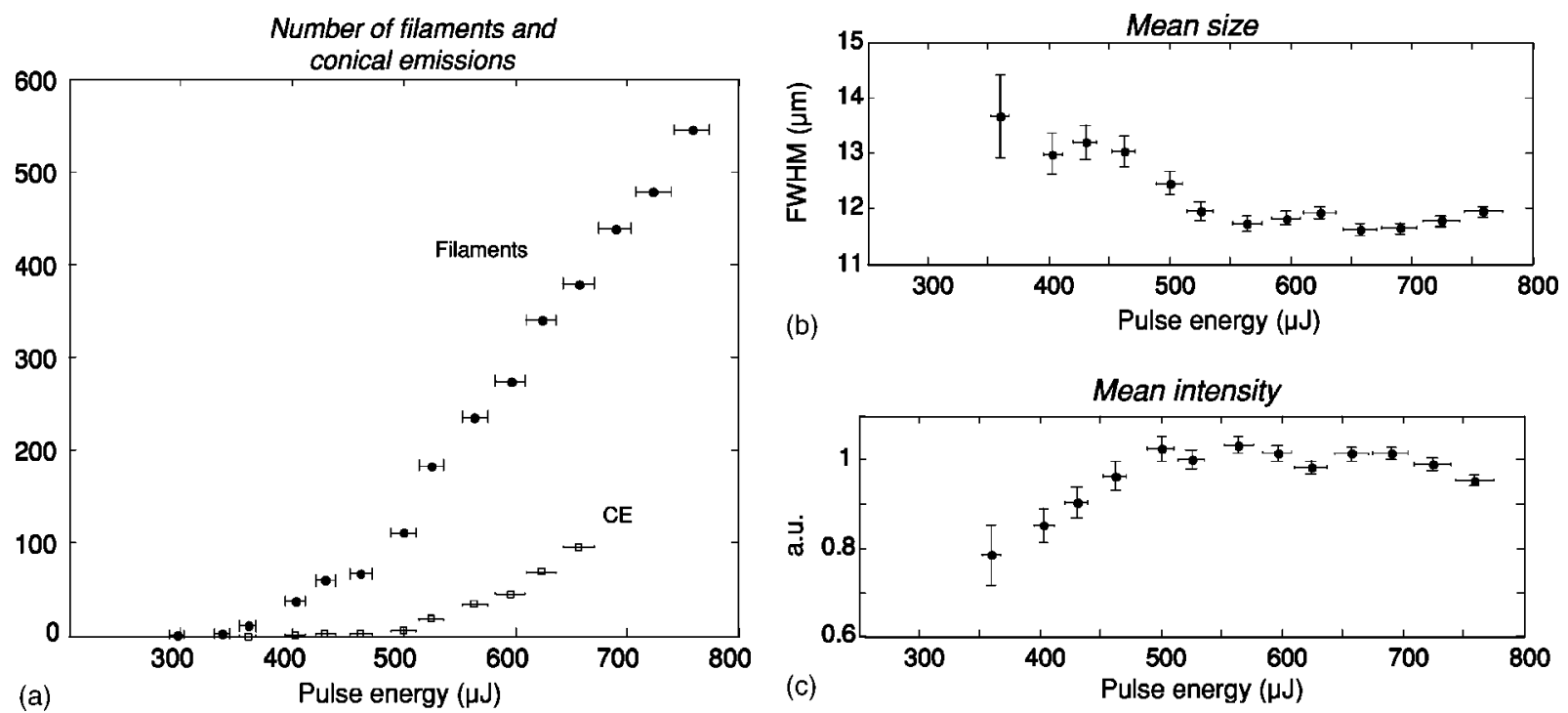

FIG. 6. Filament statistics as a function of input pulse energy. (a) Number of filaments (filled circles) and conical emissions (CE) (squares). (b) Mean size. (c) Mean intensity. A transition in the filamentation rate is observed at $0.5 \mathrm{~mJ}$.

pleted, leading to a breakdown of the phase-matching condition and an earlier transition to the filament formation process.

\section{PHASE TRANSITION IN THE RATE OF FILAMENTATION AS A FUNCTION OF INPUT ENERGY}

Figure 6(a) shows a plot of the number of filaments as a function of input power for a propagation distance of $10 \mathrm{~mm}$. There are two transition points in the rate of filament formation. For low energies no filaments are observed. As the energy increases at some point filaments begin to appear. The filamentation length depends on both the energy and the beam profile. A beam with strong modulation will break up into filaments faster, while a higher intensity will also cause the beam to break up in a shorter propagation distance. For the given beam profile, the first transition occurs when the energy is high enough for filamentation to occur in less than $10 \mathrm{~mm}$.

In the second transition we see a change in the filamentation rate [a change in the slope in Fig. 6(a)] when the input pulse energy reaches $0.5 \mathrm{~mJ}$. We carried out multiple experiments with different random input profiles. The transition to a faster filamentation rate was observed in all cases. The filamentation rate increased by a factor between 2 and 3 and the transition pulse energy was approximately the same in all cases. The transition happens when most of the filaments have reached their minimum diameter and maximum intensity. The mean filament size [Fig. 6(b)] reaches a minimum diameter of about $12 \mu \mathrm{m}$ at the transition point, while the intensity reaches a maximum near the transition [Fig. $6(\mathrm{c})]$. The energy trapped in each filament is $0.1 \pm 0.04 \mu \mathrm{J}$. Figure 6(a) also shows the number of conical waves we observed as a function of pulse energy and it clearly shows that the onset of the conical emissions coincides with the phase transition in the filamentation rate. We conclude that the conical emissions are at least partially responsible for the phase transition. During the first stage of filamentation, most of the filaments are still focusing. As the input intensity is increased, filaments release their excess energy through conical emissions before they can propagate as stable solitary waves. The radiated conical waves interfere with the background constellation and seed the generation of new filaments which appear at the intersection of the conical waves with the constellation (Fig. 7).

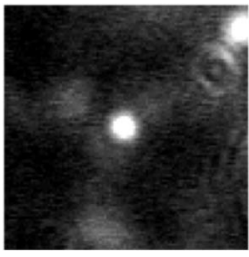

(a)

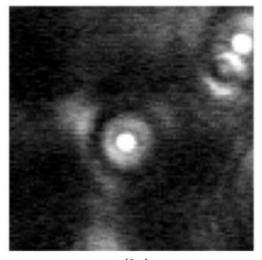

(b)

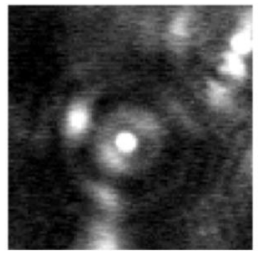

(c)

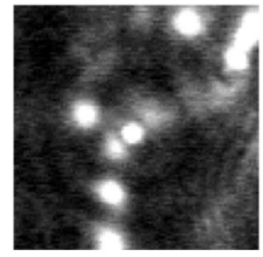

(d)

FIG. 7. Conical emission and seeding of new filaments. (a) For an input energy of $0.54 \mathrm{~mJ}$ a single filament is observed. (b) When the energy is increased to $0.64 \mathrm{~mJ}$ the filament emits conical waves before reaching the output face of the nonlinear material. (c) and (d) For higher pulse energies of 0.74 (c) and $0.86 \mathrm{~mJ}$ (d), new filaments are formed at the intersection of the conical waves with constellation. 
(a)
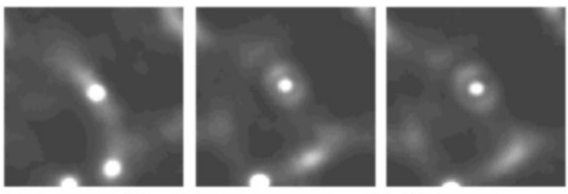

(b)
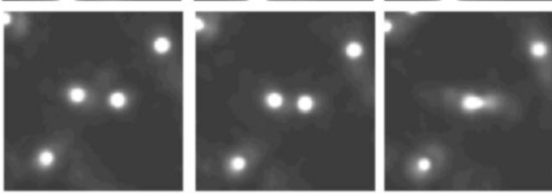

.

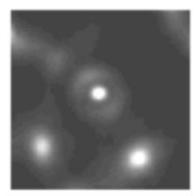

(c)
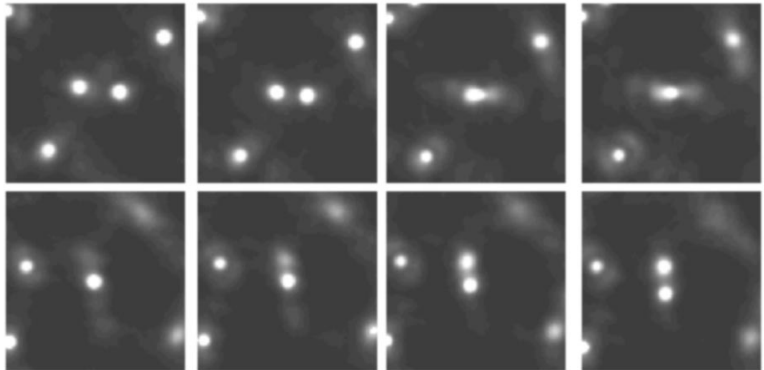

(d)
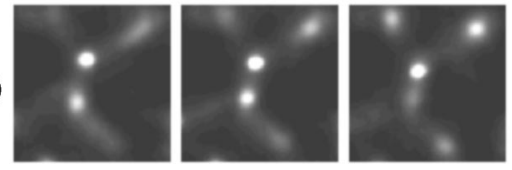
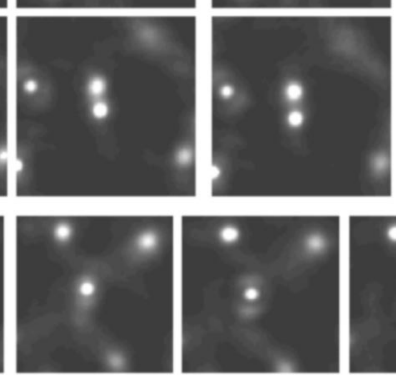

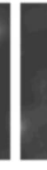

FIG. 8. Behavior of individual filaments. (a) Experimental observation of conical emission. (b) Fusion of two filaments. (c) Splitting. (d) Fusion followed by conical emission. The step in propagation distance is $0.50 \mathrm{~mm}$ for (a) and $0.25 \mathrm{~mm}$ for (b), (c), and (d).

\section{CONICAL EMISSION, FILAMENT SPATIAL SPLITTING, AND FUSION}

\section{A. Experimental results}

Figure 8(a) shows an example of conical emission for four different propagation distances at a fixed pulse energy. The filament survives and continues to propagate after the conical emission. The peak intensity is reduced by $30-50 \%$. The emission is not a continuous process. From our observations we estimate the duration of the emission to be less than 1.5 ps. Multiple conical emission was also observed in the form of a pair of rings around the filaments.

We have also measured the spectral bandwidth of the filaments before and after the conical emission. The Kerr nonlinearity causes the spectrum of the filaments to broaden as they traverse the material. We did not observe spectral broadening of the light in the constellation that is not trapped in a filament. The intensity level of the constellation is not sufficiently high to significantly broaden the spectrum of the pulse. The collapse leading to conical emission causes a sudden change in the spectrum of the filament. We deduced this by measuring the spectral content of the filaments before and after conical emissions. Specifically, the filaments were imaged onto the CCD camera using a bandpass filter with peak transmission of $60 \%$ at $805 \mathrm{~nm}$ and $34 \mathrm{~nm} \mathrm{FWHM.} \mathrm{The}$ spectrum of the pulses coming out of the laser is approximately Gaussian, centered at $800 \mathrm{~nm}$, with a FWHM of $7 \mathrm{~nm}$. Before the conical emission, the filter transmits $45 \%$ of the light trapped in the filaments. After the conical emission, the spectrum of the filament broadens and only $15 \%$ of the light is transmitted by the filter. In contrast, the spectrum of the conical waves did not broaden and was similar to that of the filaments before the conical emission. We will offer an explanation of this phenomenon in the following section.

We have observed interactions between the filaments in the form of fusion of two filaments [Fig. 8(b)] and the splitting of a single filament in two [Fig. 8(c)]. We believe the fusion is due to an attractive force, which exists if the two filaments are in phase. We attribute the splitting to filaments having excess energy and an asymmetric spatial profile. We have also observed two filaments merging and then immediately radiating [Fig. 8(d)], and filaments splitting into more than two new filaments.

\section{B. Simulations and discussion}

We have numerically calculated the propagation of a single filament including both the spatial and temporal profile of the pulse. We use a model which assumes the spatial profile of the filament is circularly symmetric:

$$
\frac{\partial A}{\partial z}=\frac{i}{2 k n_{0}} \frac{1}{r} \frac{\partial}{\partial r}\left(r \frac{\partial A}{\partial r}\right)+i k\left(n_{2}|A|^{2}\right) A+i k\left(n_{4}|A|^{4}\right) A-\frac{j \beta_{2}}{2} \frac{\partial^{2} A}{\partial T^{2}},
$$

where $A(r, z, T)$ is a function of $r, z$, and time and $r$ is measured from the center of the filament (in cylindrical coordinates).

We have simulated the propagation of a transform-limited pulse with a pulse width of $150 \mathrm{fs}$ (FWHM) for a distance of $4 \mathrm{~mm}$. The initial spatial profile is Gaussian with a FWHM of $30 \mu \mathrm{m}$ and power $P=8 P_{c r}$. The input pulse is of the form

$$
A(z=0, r, T)=\sqrt{I_{0}} \exp \left(\frac{-r^{2}}{w_{0}^{2}}\right) \operatorname{sech}\left(\frac{\mathrm{T}}{\mathrm{T}_{0}}\right) .
$$

The critical power is given by

$$
P_{c r}=\frac{\pi(0.61)^{2} \lambda^{2}}{8 n_{0} n_{2}}=1.9 \times 10^{5} \mathrm{~W} .
$$

A large input power was necessary to observe the conical emission. The pulse self-focuses into a filament and emits conical radiation after propagating for $1.5 \mathrm{~mm}$. The duration of the pulse does not change significantly during the propagation. The simulation shows that the pulse width at the center of the filament varies between 100 and $200 \mathrm{fs}$ over a propagation distance of $4 \mathrm{~mm}$, while the spatially averaged pulse width increases monotonically from 150 to $190 \mathrm{fs}$. The 


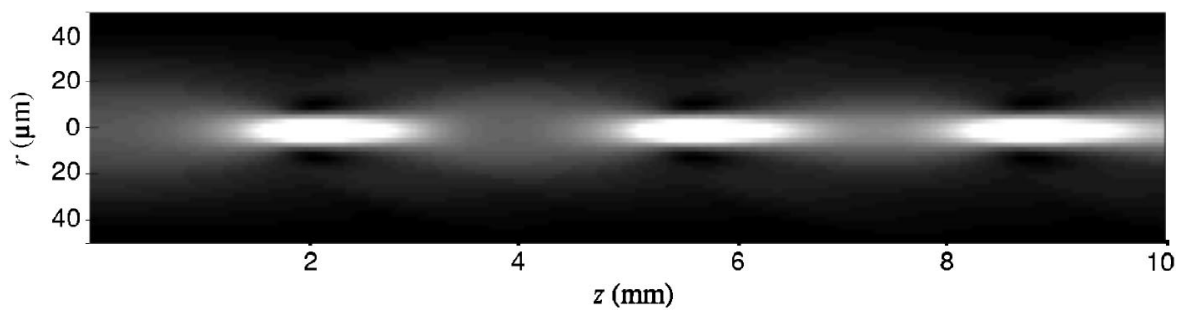

FIG. 9. Simulation for the propagation of a single filament over $10 \mathrm{~mm}$. The intensity of the filament is plotted as a function of radius (vertical scale) and propagation distance (horizontal scale). The filament is observed to periodically re-focus.

spectrum of the pulse, however, is observed to broaden significantly. The spectral bandwidth of the filament (the central part of the beam) increases from 2 to $10 \mathrm{THz}$ after $2 \mathrm{~mm}$ (right after the conical emission) and to $15 \mathrm{THz}$ after propagating for a total distance of $4 \mathrm{~mm}$. The spectral bandwitdth of the conical emission does not broaden and remains similar to the initial filament spectrum, in agreement with the experimental results obtained with the bandpass filter. The observed conical emission was, however, weaker than that observed experimentally. We believe this difference can be accounted for by the presence of the constellation and background light around the filaments. The fact that the temporal profile of the pulse does not change significantly allows us to study the conical emission in more detail ignoring spectral broadening and dispersion using the beam propagation model to numerically evaluate Eq. (3).

Before the filaments reach a steady state, the focusing is stronger for the central (brighter) part of the filament. As the filament nears its minimum radius, the outer part of the light distribution cannot keep up with the focusing and starts to diffract. We believe this is the mechanism that generates the observed conical emission rather than modulation instability. This explanation is consistent with the observed spectral composition of the beam following a conical emission. The outer part of the beam was less intense since it was not tightly focused and the spectrum was not broadened.

We have also observed in our numerical simulations an increase in the strength of the conical emission in the presence of background light. When the filament is surrounded by a uniform light distribution, the conical emission is amplified and multiple rings can be observed. We attribute this to the filaments taking in energy from the background during the self-focusing stages. This increases the amount of energy in the tails of the filament spatial distribution, which is then released through the conical emission. If the filament contains enough energy multiple rings can be generated from different parts of the spatial profile.

Simulations for a single filament show a periodic refocusing (soliton breathing [38]). We have numerically solved Eq. (3) to simulate the periodic focusing of a single filament (Fig. 9). The input beam has a Gaussian spatial profile with a $64 \mu \mathrm{m}$ diameter and power equal to five times the critical power. Figure 9 shows the filament reaching a minimum size, defocusing, and then focusing again. We did not observe this breathing behavior experimentally. In order to explain this we carried out a simulation in which the filament is surrounded by a constellation. As the input to the simulation we used a uniform beam with an intensity of 3 $\times 10^{10} \mathrm{~W} / \mathrm{cm}^{2}$ and $3 \%$ random amplitude noise. The noise is added to induce the beam to break up into a constellation. A Gaussian peak with a diameter of $64 \mu \mathrm{m}$ and an intensity $10 \%$ higher than the background was added to induce the formation of a filament at the center. Figure 10 shows the output beam profile for propagation distances from 7.5 to $11 \mathrm{~mm}$. A filament appears at the center after $8 \mathrm{~mm}$ [Fig. 10(b)] surrounded by a constellation. The filament then emits conical waves which interfere with the constellation to seed new filaments [Figs. 10(c)-10(e)]. Finally the original filament fuses with one of the new filaments [Figs. 10(d)-10(h)]. In this case the periodic refocusing is not observed since the presence of the constellation breaks the symmetry. The breathing behavior can be thought of as a
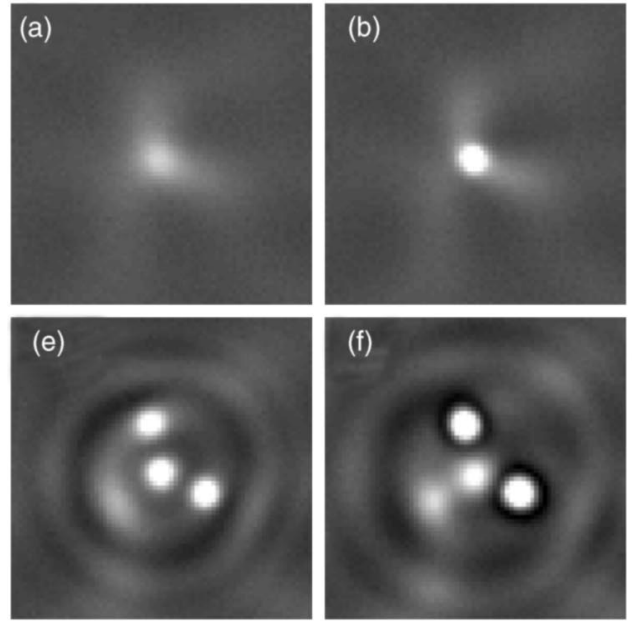
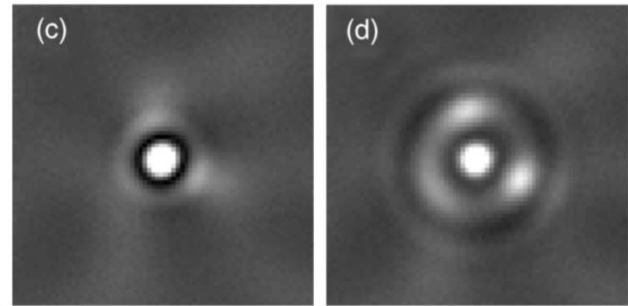

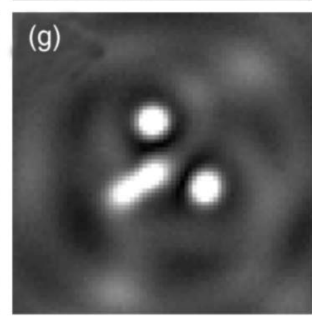

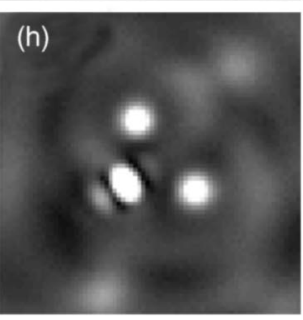

FIG. 10. Simulated filament propagation in the presence of a noisy background. (a)-(c) The filament focuses and emits conical waves. (d) and (e) The conical waves seed the formation of new filaments on the constellation. (f)(h) The original filament is attracted and fuses with a new filament. The propagation distance from (a) through (h) is from 7.5 to $11 \mathrm{~mm}$ in steps of $0.5 \mathrm{~mm}$. 
periodic failed attempt at a conical emission. The presence of the background constellation modifies this behavior when the emitted light constructively interferes with the constellation and induces the generation of new filaments. The new filaments also affect the propagation of the original filament.

\section{SUMMARY}

We have studied in detail the filamentation process in $\mathrm{CS}_{2}$ and we have identified a number of interesting behaviors. The formation of a constellation precedes the filamentation stage. We have observed a unique form of conical emission which appears to be the dominant mechanism for filaments with excess energy to relax to their steady-state level. We have also observed a phase transition in the filamentation rate at the onset of conical emission from the filaments. We attribute the change in the filamentation rate to interactions between the constellation and the conical emissions. The spectral bandwidth of the filaments was observed to increase after conical emission, while the spectrum of the conical waves did not broaden. Finally we observed fusing and splitting of filaments both experimentally and numerically.

\section{ACKNOWLEDGMENTS}

This work was sponsored by the Engineering Research Centers Program of the National Science Foundation under Award No. EEC-9402726 and the Defense Advanced Research Projects Agency (DARPA) Center for Optofluidic Integration.
[1] F. Shimizu, Phys. Rev. Lett. 19, 1097 (1967).

[2] R. R. Alfano and S. L. Shapiro, Phys. Rev. Lett. 24, 592 (1970).

[3] A. J. Campillo, S. L. Shapiro, and B. R. Suydam, Appl. Phys. Lett. 23, 628 (1973).

[4] V. I. Bespalov and V. I. Talanov, JETP Lett. 3, 307 (1966).

[5] A. J. Campillo, S. L. Shapiro, and B. R. Suydam, Appl. Phys. Lett. 24, 178 (1974).

[6] L. Berge et al., Phys. Rev. Lett. 92, 225002 (2004).

[7] X. H. Ni et al., IEEE J. Sel. Top. Quantum Electron. 10, 1229 (2004).

[8] A. Barthelemy, S. Maneuf, and C. Froehly, Opt. Commun. 55, 201 (1985).

[9] M. Segev et al., Phys. Rev. Lett. 68, 923 (1992).

[10] R. Boyd, Nonlinear Optics (Academic Press, San Diego, 2003).

[11] M. Saffman, G. McCarthy, and W. Krolikowski, J. Opt. B: Quantum Semiclassical Opt. 6, S397 (2004).

[12] G. G. Luther et al., Opt. Lett. 19, 789 (1994).

[13] G. G. Luther et al., Opt. Lett. 19, 862 (1994).

[14] J. K. Ranka, R. W. Schirmer, and A. L. Gaeta, Phys. Rev. Lett. 77, 3783 (1996).

[15] A. Brodeur and S. L. Chin, J. Opt. Soc. Am. B 16, 637 (1999).

[16] B. D. Paul et al., Phys. Rev. A 59, 4784 (1999).

[17] E. T. J. Nibbering et al., Opt. Lett. 21, 62 (1996).

[18] O. G. Kosareva et al., Opt. Lett. 22, 1332 (1997).

[19] J. P. Gordon, Opt. Lett. 8, 596 (1983).
[20] G. I. Stegeman and M. Segev, Science 286, 1518 (1999).

[21] M. Shalaby, F. Reynaud, and A. Barthelemy, Opt. Lett. 17, 778 (1992).

[22] H. Schroeder and S. L. Chin, Opt. Commun. 234, 399 (2004).

[23] R. A. Ganeev et al., Appl. Phys. B: Lasers Opt. 78, 433 (2004).

[24] A. Piekara, IEEE J. Quantum Electron. QE-2, 249 (1966).

[25] S. C. Abbi and H. Mahr, Phys. Rev. Lett. 26, 604 (1971).

[26] A. Samoc, Appl. Phys. Lett. 94, 6167 (2003).

[27] M. Falconieri and G. Salvetti,Appl. Phys. B: Lasers Opt. 69, 133 (1999).

[28] K. Ishikawa, H. Kumagai, and K. Midorikawa, Phys. Rev. E 66, 056608 (2002).

[29] C. Courtois et al., Phys. Plasmas 8, 3445 (2001).

[30] N. Akozbek et al., Phys. Rev. E 61, 4540 (2000).

[31] L. V. Keldysh, Sov. Phys. JETP 20, 1307 (1965).

[32] P. K. Kennedy, IEEE J. Quantum Electron. 31, 2241 (1995).

[33] C. P. Safvan et al., J. Phys. B 29, 3135 (1996).

[34] P. K. Kennedy et al., IEEE J. Quantum Electron. 31, 2250 (1995).

[35] Q. Feng et al., IEEE J. Quantum Electron. 33, 127 (1997).

[36] G. Fibich, Phys. Rev. Lett. 76, 4356 (1996).

[37] G. P. Agrawal, Nonlinear Fiber Optics (Academic Press, San Diego, 1995).

[38] D. E. Pelinovsky, V. V. Afanasjev, and Y. S. Kivshar, Phys. Rev. E 53, 1940 (1996). 